\title{
Indeks osobowy
}

A

Adler Filip 90

Adler Friedrich 14, 15, 20

Albrechtau Jan 103

Aleksandrowicz Wiktor 164

Allen von (rodzina) 16

Anger Siegfried, dr 30

Antoni Padewski, św. 119

Arystoteles 117, 145

Attavante degli Attavanti 116, 117, 118

B

Bartel Bronisław 176, 177, 178, 187, 348 (il. 3)

Batory Andrzej 140

Batory Balthazar 140

Bernardyn, św. 119

Berzeviczy Martin de 140

Bewthau Thomas 103

Biedowicz Jan 181, 182, 187, 352 (il. 9)

Bielke Ture 140, 323 (il. 3)

Blemke Eduard 138, 139, 140, 147, 148, 325 (il. 5)

Blocke Philip van der 141

Blocke Willem van der 139, 140, 141, 150

Blumenau Michael 103

Boccardi Giovanni 116, 117, 120

Boccardino il Vecchio 117

Bodeck Valentin von 139, 147, 148

Bogusław I, książę pomorski 73, 88

Boissard Jean-Jacques 144, 147, 328 (il. 8), 330 (il. 10)
Bolesław Chrobry 87

Bonawentura, św. 103

Borucki Kazimierz 186

Botticelli Sandro 116

Bottone Bernardus Parmensis de 108, 112

Bouts Dirk 116

Branconi Antonio von 119

Branconi Karolina von 119, 123

Brandes Dorothea 145

Brandes Johann 139, 145, 329 (il. 9)

Brandes (rodzina) 140, 141, 146, 147

Brederlow Fryderyk Bonawentura von 114, 119, 122, 123

Brejska-Malessina Janina 165

Brzęczkowski Stanisław 175

Burhardt Stefan, dr 96

Bünau Günther von, bp samb. 102

C

Canaletto 181

Cennini Cennino 127

Chiaromonte Izabela 118

Chierico Francesco d'Antonio del 117, 118

Chmura Piotr 177, 179, 187, 346 (il. 1), 350 (il. 5)

Chrystian, bp 52, 61

Chytraeus Nathan 148

Ciechanowski Franciszek 153, 154, 156, 157, 159

Ciechanowski Piotr 155, 158, 331 (il. 1), 333 (il. 5)

Clare Jan, bp samb. 102 
Clercx de Ghele Jacobus 101

Cock Hieronymus 139

Comestor Piotr 99, 103, 299 (il. 3)

Cremona Girolamo da 117

Curtius Ernst 15

Cyceron 86

Czaplicki Roman 167, 184

\section{D}

Dawid, król 120, 129, 132, 314 (il. 4)

Dobilstein Wikbold, bp chełmiński 101, 102

Dobrowolski Tadeusz 163, 167, 170, 186

Dohna Christopher von 140

Dołżycki Leon 177, 178, 187, 349 (il. 4)

Drapiewski Władysław 182, 186

Dufy Raoul 167

Durand Wilhelm 104

Durek Wojciech Aleksander 185, 187

Durster Henryk 108

\section{E}

Ellicot Thomas 219

Esken Frantz 143

Esken Margaretha 143

Eugeniusz, św. 120

Evans Oliver 219

Ezechiel 147, 148

\section{F}

Faczyński Marian 181, 182, 187

Fahlberg Arthur 31

Falkenau Andreas 102

Fałat Julian 162, 171, 172, 173, 337 (il. 1)

Fauth Wilhelm 31

Federigo da Montefeltre, książę Urbino 117

Fidanza Stanisław 185

Floris Cornelis 139

Francken Ambrosius 146

Frisius Caspar 138

Frodl Walter 192, 193, 194, 205

Fryderyk I Barbarossa 88

Fryderyk Wilhelm 86

Frydrych Władysław 182, 183, 187
G

Gadziewski Auguste 30

Gajewski Franciszek 181, 186, 187, 352 (il. 8)

Galimski Władysław 179, 180, 187

Gall Anonim 87

Gerlachus 127

Gęstwicki Brunon 153, 155, 156, 162, 163, 164, 165, 173, 185, 187, 331 (il. 1), 334 (il. 6), 341 (il. 6),

Gęstwicki Feliks Paweł 163, 171, 185, 187, 341 (il. 7)

Gilly David 13

Gilly Friedrich 13

Górski Karol, prof. 34, 132

Graser Starszy Konrad 143, 149

Gromek Wacław 181, 182, 187

Groote Geert 100, 101, 300 (il. 4)

Gros Eugeniusz 162, 172, 173, 185, 187, 339 (il. 3)

Groskurd Alwina 13

Grottkau Jan de 102

Grzegorz Wielki, św. 149

Grzymisław, książę 84

Gustaw Adolf 102

\section{H}

Haecht Willem van 146

Hagen Gotthilf 13

Han Herman 74, 91, 92

Hannytkiewicz Adam 169

Hawryłkiewicz Jan 184, 187

Heise Johannes 23, 25, 32, 51, 59

Heuer Reinhold 65, 68, 69

Hindersin Johann Caspar 17

Hiob 146

Hirschfeld Gustav 15

Hohenzollern Albrecht, książę 96, 107

\section{I}

Izajasz 129

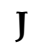

Jacewiczowa Zofia 162, 173, 338 (il. 2)

Jacobi Hans 42 
Jan Ewangelista, św. 103

Jan Gwalbert, św. 120

Jarosiewicz Janusz 164

Jeroschin Mikołaj von 95

Jezus Chrystus 82, 85, 103, 125, 126, 129, 130, 131, 147

Jędrkiewiczowa Jadwiga 165, 185, 187

Jędrzejewski Aleksander 184

John Christina Dorotea (z d. Kerstein) 25

John Christina Elisabeth (z d. Kerstein) 25

John Johann Thomas 25

Joseph Hermann 101

Justynian 80

\section{K}

Kalizer Nicolaus 102

Karniej Edward 164, 165, 167, 171, 173, 345 (il. 11)

Kempen (rodzina) 143

Kiersnowski Stefan 183, 187, 355 (il. 12)

Klara, św. 119

Kochanowska Ausgusta 165

Kolber Oskar 207, 208, 211

Kolwitz Emil 157, 159

Kolwitz Ludwig 156

Kolwitz (rodzina) 156, 157, 159

Konitzer Franciszek 179, 180, 187

Konstantyn Wielki 82

Korn Richard, dr inż. 31

Korwin Maciej 117, 118

Kowarski Felicjan Szczęsny 162, 173, 338 (il. 2)

Kozłowski Józef 165

Krassowski Feliks 166, 173, 184, 187, 343

(il. 9), 356 (il. 13)

Krystoszek Wacław 182, 187, 346 (il. 1)

Kuhn Włodzimierz 166, 173, 344 (il. 10)

Kuhnd Karl 28

Kujawa Marian 186

L

Lange Roderyk 208, 211

Lasocki Czesław, hr. 96

Laszenko Aleksander 169, 171

Lessen Jan 102
Lewandowski Bernard 183, 187

Lewański Bolesław 176, 177, 187, 347 (il. 2)

Lignau Franciszek Ernest 71, 76, 85, 88, 89, 91, 289 (il. 1)

Lippi Filippo Fra 116

Luter Marcin 149

Luter z Brunszwiku 99

\section{M}

Magdańska-Dąbrowska Halina 183, 187

Makojnik Wiesław Bazyli 167

Malewski Zygmunt 177

Małkowski Witold 166

Markant Jean 116

Matejko Jan 169

Mateusz Ewangelista, św. 145

Matylda, księżna 84

Mazurek Ignacy 162, 164, 165, 168, 171, 173, 174, 185, 339 (il. 4)

Medici Laudomia de 116, 117

Medici Wawrzyniec de 118

Medyceusze (rodzina) 117

Memling Hans 116

Mestwin I (Mszczuj, Mściwój, Mściwoj) 72, $82,84,90$

Michał, bp włock. 76, 85

Mieszko III 84

Mikołaj (z Myry), św. 79, 80, 81

Miller Stanisław 164

Mitera Kazimierz 166

Modlibowski Aleksander 186

Mokrzycki Tadeusz 182, 187, 353 (il. 10)

Mokwa Marian 185

Mondral Karol 177, 179, 185, 187

Montucci Albert 119, 122

Möllen Kilian von 63

Möller Anton 17

Mszczuj $\rightarrow$ Mestwin

Mściwoj (Mściwój) $\rightarrow$ Mestwin

Mściwoj II, książę 90

Myszkorowski Zygmunt 186

\section{$\mathrm{N}$}

Neisser (rodzina) 19, 149

Neuwirth Joseph 28 
Niesiołowski Tymon 165, 168, 169, 171,

O

Oelsnitz Anna Ida Ernestine Julie 24

Oelsnitz Ernest von der 34

Otton III 80

\section{$\mathbf{p}$}

Paradin Claude 144, 147

Pessina (rodzina) 119

Peters Ernst 151-153, 155-159

Peters Marianna 156

Pictaviensis Piotr 103

Pindelski Leszek164

Piotrowski Antoni Maksymilian 171

Platon 118, 145

Podlaszewski Wojciech 164, 185, 187

Priskos, św. 80

Procajłowicz Antoni S. 176, 177, 178, 187

Prokopiusz z Cezarei 80

Prokulf, św. 119

Przeradzka-Jędrzejewska Jadwiga 184

Przybył Eugeniusz 162, 165, 185, 187, 340 (il. 5)

Przybyłowa Janina 165, 169

Przybyłówna Jadwiga (Jadzia) 162, 340 (il. 5)

Pseudo-Arystoteles 117

Pseudo-Hipokrates 118

Puffke Marian 164

\section{R}

Raciniewski Leon 167

Radam Bartholomäus de, bp samb. 102

Reparata, św. 119

Reusner Nicolaus 148

Rickert Heinrich 192

Riegl Alois 192

Riga Piotr 103

Romulus, św. 120

Rosselli Francesco 117

Rudyger, opat cyst. w Oliwie 74, 81, 88, 90, 91, 92

Ruperti Andreas 103

Rupniewski Jerzy 165, 175, 176, 181, 186, 187, 351 (il. 7)
Rysiewski Bronisław 167

\section{S}

Saegerus Joachim 148

Salendorf Friedrich 101

Salomon, król 132, 314 (il. 4)

Sambor I, książę 72

Sambor II, książę 84

Samlicki Marcin 171

Samson 115

Schachmann (rodzina) 143

Schmid August 24

Schmid Bernhard 9, 21-35

Schmid Carolina Elisa Augusta 25

Schmid Christian August Emil 24

Schmid Christina Elisabeth (z d. John) 25

Schmid Friedrich Ludwig 24

Schmid Henriette Franziska Emma 25

Schmid Henriette Friedericke (z d. Prange) 24

Schmid Johann Carl 25

Schmid Johann Gotlieb August 25

Schmid Johann Zacharias 25

Schmid Ludwig 25

Schulze Eryka 165

Schulze-Koeper Anna 165

Schütz Casper 72

Sehring Bernhard 152

Sieński Franciszek 176, 185

Sobieska Jadwiga 209

Sobieski Marian 209

Soest von (rodzina) 16

Spieker Paul Emanuel 15

Stankiewiczówna Zofia 171

Steinborn Helena 165

Steinbrecht Alwina (z d. Groskurd) 13

Steinbrecht Conrad Emanuel 9, 14-20, 22, $26,27,28,2932,33,41,51,53,61,257-266$ (il. 1- il. 12)

Steinbrecht Edward 13

Stotten Anna 143

Stroband (rodzina) 137-150, 321 (il. 1), 324 (il. 4), 327 (il. 7)

Stroband Christian 137, 140, 143, 327 (il. 7) 
Stroband Heinrich 141, 144, 149

Stroband Johann 137, 140, 141, 143, 327 (il. 7)

Stüler Friedrich August 13

Subisław I (Senior) 71, 72, 73, 75, 76, 77, 81, 82, 84, 85, 86, 89, 90, 91

Suchanek Antoni 186

Szczeblewski Wacław 180, 185

Szmaj Stefan 182, 187

\section{Ś}

Śliwińska-Kapturkiewicz Elżbieta 176, 185

Święcicki Józef 156

Świętopełk, książę pomorski 72, 73, 75, 76, 82, 85, 86, 88, 89, 90, 91

\section{$\mathbf{T}$}

Taljański Albert Lewy 176, 179, 180, 187

Tarkowski Tadeusz 186

Teofil (Mnich) 126, 127

Terlecki Alfred 171

Torelli Filippo di Matteo 117, 118

Triebler Piotr 175, 346 (il. 1)

Trisner Martin 143, 144, 145, 147, 150

Turwid Marian 175, 182, 183, 187, 354 (il. 11)

\section{U}

Unelf (Wnelphus, Unolfus, Unelfus, Onolf), bp włock. 83, 85

Urszula, św. 101, 116

\section{V}

Vante di Gabriello di Vante $\rightarrow$ Attavante degli Attavanti
Volmar Erich 29

Vrelant Wilhelm 100, 116

Vries Hans Vredeman de 139

\section{W}

Wachowiak Stanisław 168

Wallenrod Konrad von 19

Waluk Kazimierz 164, 173

Weidner Fritz 153

Werle von (rodzina)16

Wesolowski Andreas 27

Weyden Rogier van der 116

Węgrzyn Stanisław 184

Wierix Hieronymus 146

Wiktor, św. 104

Willy Konrad, ks. 29

Winnicki-Radziewicz Aleksander 186

Witkiewicz Stanisław Ignacy 168, 171

Witt Martin 30

Władysław IV 196

Wojciechowski Stefan 164

Wolfgang, św. 26, 29, 35

Wyczółkowski Leon 171

Wysocki Jan 176

\section{Z}

Zacholski Władysław 186

Zelek Ignacy 163, 169, 185, 187, 342 (il. 8)

Zirenberg (rodzina) 143

Zwinisława, księżna 84

\section{$\dot{\mathrm{Z}}$}

Żyron, palatyn mazowiecki 84 